\title{
GENERICITY OF OBSERVABILITY OF CONTROL-AFFINE SYSTEMS
}

\author{
M. BALDE AND P. JOUAN
}

\begin{abstract}
For smooth or real-analytic single-input, control-affine, nonlinear systems, with at least two ouputs, observability for any input of a given class is generic. This class can be either the class of inputs bounded with their derivatives up to a certain order, or the class of polynomial inputs with bounded degree.
\end{abstract}

\section{INTRODUCTION}

In this paper we deal with genericity of observability of control-affine systems of the form:

$$
\Sigma=\left\{\begin{array}{l}
\dot{x}=f(x)+u_{0} g(x) \quad x \in X, \\
y=h(x)
\end{array} \quad u_{0} \in \mathbb{R}\right.
$$

where the state space $X$ is compact and $d$-dimensional.

Such a single-input p-outputs system is said to be observable for an input $t \mapsto u_{0}(t)$ if any two initial states are distinguished by the outputs (corresponding to a given input) on any time interval, i.e. if:

$$
\begin{gathered}
\forall x_{1} \neq x_{2} \in X, \quad \forall T>0, \quad \exists \tau \in[0, T[\text { s.t. } \\
h\left[x_{1}(\tau)\right] \neq h\left[x_{2}(\tau)\right],
\end{gathered}
$$

where $x_{i}(t)$ is the solution of the dynamical system for the input $t \mapsto u_{0}(t)$ and the initial condition $x_{i}(0)=x_{i}$.

This definition of observability is not the standard one that was proposed by Hermann and Krener in [10]. But our definition is justified by the genericity results at least when there are more outputs than inputs. It is used in $[7],[13]$ and $[16]$.

In fact, we deal with the stronger property of differential observability: a $C^{k}$-input being given, the system $\Sigma$ is said to be differentially observable for this input if, for an integer $k^{\prime} \leq k$, the mapping which associates to the state the outputs together with their $k^{\prime}$ first derivatives is an embedding. This definition permits the use of transversality tools, that are involved in order to prove the following results:

M. Balde: Laboratoire de Mathématiques, AMS-LMI, UPRES-A CNRS 6085, INSA de Rouen, BP08, Place Emile Blondel, 76131 Mont-Saint-Aignan cedex, France. E-mail: balde@lmi.insa-rouen.fr.

P. Jouan: Laboratoire de Mathématiques, AMS-LMI, UPRES-A CNRS 6085, Place Colbert, 76131 Mont-Saint-Aignan cedex. France. E-mail: Philippe. Jouan@univ-rouen.fr.

Received by the journal May 14, 1997. Revised March 31, 1998. Accepted for publication June 1, 1998.

(C) Société de Mathématiques Appliquées et Industrielles. Typeset by LATEX. 
Theorem 3.2. Fix $\mathbf{p} \geq \mathbf{2}$. The set of systems with $p$ outputs which are differentially observable for any $C^{2 d}$-input is residual in the set of all $C^{r}$ systems, for $r \in \mathbb{N} \cup\{\infty, \omega\}$ large enough.

Theorem 3.3. A bound $B>0$ being given, the set of systems that are differentially observable for any $C^{2 d}$-input $t \mapsto u_{0}(t)$ such that $\left|\frac{d^{2} u_{0}}{d t^{2}}(0)\right| \leq$ $B$ is open and dense in the set of all $C^{r}$-systems, for $r \in \mathbb{N} \cup\{\infty, \omega\}$ large enough, and for $\mathbf{p} \geq \mathbf{2}$.

Analogous statements are proved in [7], [8] for general nonlinear systems. Notice, however, that since the interior of the subset of control-affine systems is empty in the set of all non-linear systems, our results cannot be deduced from those of [7], [8].

In the statement of the above theorems, one can notice the requirement $p \geq 2$. This is in fact the general requirement that the number of ouputs is strictly larger than the number of inputs, which is also made in [7], [8]. If the number of ouputs is equal to the number of inputs the theorems are false as it is shown in $[6]$.

In section 5 we prove the following result: Theorem 5.1. The set of systems that are differentially observable for any polynomial input whose degree is smaller than a given bound contains an open and dense subset of the set of all $C^{r}$-systems, for $r \in \mathbb{N} \cup\{\infty, \omega\}$ large enough, and for $\mathbf{p} \geq \mathbf{2}$.

The proof of this theorem is based on a method of compactification of the inputs, that was used for the first time by the second author in the case of general non-linear systems (see [14], [15]).

One of the main interests of differential observability for all inputs whose derivatives belong to a bounded set is the possibility of constructing nonlinear observers. We do not give this construction here, because it follows the same line as the construction made for general non linear-systems and can be found in $[6]$ or [7]. The computations are particularly detailed in [16] where it is moreover shown that differential observability is a sufficient but not necessary condition for the possibility of the constructing of nonlinear observers.

To finish we want to recall another result proved in the uppermentionned paper [7], and that is immediatly available for our particular systems:

Theorem 5.1 of [7]. For an analytic system $\Sigma$, the following properties are equivalent:

- $\Sigma$ is observable for any $C^{\omega}$-input

- $\Sigma$ is observable for any $L^{\infty}$-input.

For the sake of clarity, we have chosen to deal with single-input systems only ; but our results can be rather easily extended to multi-input controlaffine systems assuming that the number of ouputs is strictly larger than the number of inputs.

For systems whose state manifold is not compact, similar results are certainly true in the Whitney topology. Nevertheless, the observer design problem is well posed only if the state space is compact or when restricting to a compact subset (see for instance [16]). For this reason we consider the compact case only.

ESAIM: COCV, OCTOBER 1998, VoL. 3, 345-359 


\section{DEFINITIONS AND NOTATIONS}

Let $X$ be a $d$-dimensional, compact, connected $C^{\infty}$-manifold.

Let $h: X \longrightarrow \mathbb{R}^{p}$ be a $C^{r}$-mapping and $f, g$ two $C^{r}$-vector-fields on $X$, where $r \in \mathbb{N} \cup\{\infty\}$. We consider a single-input, p-outputs system $\Sigma=$ $(h, f, g)$ on $X$ defined by:

$$
\Sigma=(h, f, g)=\left\{\begin{array}{ll}
\dot{x}=f(x)+u_{0} g(x) & x \in X, \\
y=h(x) & y \in \mathbb{R}^{p} .
\end{array} u_{0} \in \mathbb{R}\right.
$$

The set $C^{r}\left(X, \mathbb{R}^{p}\right)$ (resp. the set $\Gamma^{r}(T X)$ of $C^{r}$-vector-fields on $X$ ) will be denoted by $H^{r}$ (resp. $\Gamma^{r}$ ) and endowed with the $C^{r}$-topology. $S^{r}$ will stand for the set $H^{r} \times\left(\Gamma^{r}\right)^{2}$ of $C^{r}$-systems. For $r<\infty, H^{r}, \Gamma^{r}$ and $S^{r}$ have the natural structure of Banach spaces (see [1]).

In what follows, the integer $r$ will be supposed to be large enough in order to perform all considered operations.

The $k^{t h}$ dynamical extension of the vector-field $f+u_{0} g$ is the vector-field $F^{k}$ on $X \times \mathbb{R}^{k}$ controled by $u_{k}$ and defined by:

$$
\begin{aligned}
& F^{k}\left(x, u, u_{k}\right)=f(x)+u_{0} g(x)+\sum_{i=0}^{k-1} u_{i+1} \frac{\partial}{\partial u_{i}}, \\
& \text { where } \quad u=\left(u_{0}, u_{1}, \ldots, u_{k-1}\right) .
\end{aligned}
$$

A system $\Sigma=(h, f, g) \in S^{r}$ being given and for $1 \leq k \leq r+1$ we define the mapping $R_{k, \Sigma}$ by:

$$
\begin{gathered}
R_{k, \Sigma}: X \times \mathbb{R}^{k} \longrightarrow \mathbb{R}^{k p} \\
(x, u) \mapsto\left(h(x), L_{F^{k}} h(x, u), \ldots, L_{F^{k}}^{k-1} h(x, u)\right) .
\end{gathered}
$$

REMARK 2.1.

1. $R_{k, \Sigma}$ depends on $u=\left(u_{0}, u_{1}, \ldots, u_{k-1}\right)$ but not on $u_{k}$.

2. Let $t \longmapsto u_{0}(t)$ be an input which verifies $\frac{d^{i} u_{0}}{d t^{i}}(0)=u_{i}$ for $i=0 \cdots k-1$. It is clear that

$$
R_{k, \Sigma}(x, u)=\left(h(\phi(t)), \frac{d}{d t}[h(\phi(t))], \ldots, \frac{d^{k-1}}{d t^{k-1}}[h(\phi(t))]\right)_{t=0},
$$

where $t \longmapsto \phi(t)$ is the solution of $\dot{x}=f(x)+u_{0}(t) g(x)$ passing by the initial condition $\phi(0)=x$.

We will also use the suspension mapping $S R_{k, \Sigma}$, which is merely:

$$
S R_{k, \Sigma}(x, u)=\left(R_{k, \Sigma}(x, u), u\right) \in \mathbb{R}^{k p} \times \mathbb{R}^{k}
$$

Since $X$ is a compact manifold, $S R_{k, \Sigma}$ is always a proper mapping. We are interested in systems $\Sigma$ for which $S R_{k, \Sigma}$ is an embedding, at least when restricted to $X \times U$ where $U$ is a subset of $\mathbb{R}^{k}$, for the following reasons:

1. If the restriction of $S R_{k, \Sigma}$ to $X \times U$, is one-to-one, then, according to remark $2.1(2), \Sigma$ is observable for any input whose $(k-1)$ first derivatives belong to $U$.

2. The fact that $S R_{k, \Sigma}$ is additionally an embedding is a sufficient condition to construct observers. 


\section{TRANSVERSALiTy RESULTS}

\subsection{Statement of the Results}

The following theorems holds only for $\mathbf{p} \geq \mathbf{2}$, i.e. if the number of ouputs is strictly larger than the number of inputs.

THEOREM 3.1. The set of systems $\Sigma$ such that $S R_{2 d, \Sigma}$ is an immersion contains an open and dense subset of $S^{r}$ (for $r \in \mathbb{N} \cup\{\infty\}$, large enough).

THEOREM 3.2. The set of systems $\Sigma$ such that $S R_{2 d+1, \Sigma}$ is an embedding is residual hence dense in $S^{r}$ (for $r \in \mathbb{N} \cup\{\infty\}$, large enough).

Theorem 3.3. Let $B>0$ and $I_{B}=[-B, B]$. The set of systems $\Sigma$ such that the restriction of $S R_{2 d+1, \Sigma}$ to $X \times\left(I_{B}\right)^{2 d+1}$ is an embedding is open and dense in $S^{r}$ (for $r \in \mathbb{N} \cup\{\infty\}$, large enough).

\subsection{SOME REMARKS}

1. We recall the following definition: a subbundle $B$ of a vector bundle $E$ is called partially semi-algebraic (PSA) if its typical fiber is a semialgebraic subset of the typical fiber of $E$ (see [4],[1],[17],[11]). Standard properties of PSA subbundles are used in the following proofs.

2. We denote by $\oplus$ the fiber product over $X$. Thus the bundle of $k$-jets of systems is the bundle:

$$
J^{k} S=J^{k} H \oplus J^{k} \Gamma \oplus J^{k} \Gamma .
$$

3. The mapping $R_{k, \Sigma}$ (resp. $T_{X} R_{k, \Sigma}$ ) depends only on the $(k-1)$ )-jet

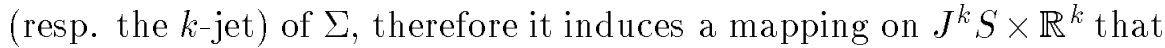
is also denoted by $R_{k, \Sigma}$ (resp. $\left.T_{X} R_{k, \Sigma}\right)$.

4. If a point $\left(x, u_{0}\right)$ is a singularity of the system $\Sigma$, i.e., if $f(x)+$ $u_{0} g(x)=0$, we will sometimes denote by $(C, A)$ the linearized system $\left(d h(x), T_{X}\left(f(x)+u_{0} g(x)\right)\right)$.

\subsection{Proof of the Theorem 3.1}

Let $\hat{B}(k)$ denote the subset of $J^{k} S \times \mathbb{R}^{k}$ of all $\left(j_{x}^{k} \Sigma, u\right)$ such that rank $\left[T_{X} R_{k, \Sigma}(x, u)\right]<d$. We consider the following partition of $\hat{B}(k)$ :

$$
\hat{B}(k)=\hat{B}_{1}(k) \cup \hat{B}_{2}(k) \cup \hat{B}_{3}(k)
$$

where:

- $\left(j_{x}^{k} \Sigma, u\right) \in \hat{B}_{1}(k) \Longleftrightarrow f(x)+u_{0} g(x) \neq 0$

- $\left(j_{x}^{k} \Sigma, u\right) \in \hat{B}_{2}(k) \Longleftrightarrow\left\{\begin{array}{l}f(x)+u_{0} g(x)=0 \\ \text { and the linearized system } \\ \left(d h(x), T_{X}\left(f(x)+u_{0} g(x)\right)\right) \text { is not observable. }\end{array}\right.$

- $\left(j_{x}^{k} \Sigma, u\right) \in \hat{B}_{3}(k) \Longleftrightarrow\left\{\begin{array}{l}f(x)+u_{0} g(x)=0 \\ \text { and the linearized system } \\ \left(d h(x), T_{X}\left(f(x)+u_{0} g(x)\right)\right) \text { is observable. }\end{array}\right.$

$\hat{B}(k), \hat{B}_{1}(k), \hat{B}_{2}(k), \hat{B}_{3}(k)$ and their respective projections $B(k), B_{1}(k)$, $B_{2}(k), B_{3}(k)$ into $J^{k} S$ are PSA.

ESAIM: COCV, OCTOBER 1998, VoL. 3, 345-359 
3.3.1. $\operatorname{Codim}\left(B_{1}(k)\right)=k(p-1)+1-d$. Let

$$
O_{1}=\left\{\left(j_{x}^{k} \Sigma, u\right) \in J^{k} S \times \mathbb{R}^{k}, \quad f(x)+u_{0} g(x) \neq 0\right\} .
$$

Notice that $O_{1}$ is an open subset of $J^{k} S \times \mathbb{R}^{k}$. Let $\mu_{1}$ be the mapping:

$$
\begin{aligned}
\mu_{1}: \quad O_{1} & \longrightarrow\left(T^{*} X\right)^{\oplus k p} \\
\left(j_{x}^{k} \Sigma, u\right) & \longmapsto T_{X} R_{k, \Sigma}(x, u) .
\end{aligned}
$$

The mapping $\mu_{1}$ is a submersion because, by Lemma 6.2 , the linear mapping $j_{x}^{k} h \mapsto T_{X} R_{k, \Sigma}(x, u)$ is surjective as soon as $f(x)+u_{0} g(x) \neq 0$. Notice that $\hat{B}_{1}(k)$ is the pullback by $\mu_{1}$ of the set of elements of $\left(T^{*} X\right)^{\oplus k p}$ whose rank is less than $d$. By the product of coranks theorem (see [9]), the codimension of this set is $(d-(d-1))(k p-(d-1))=k p+1-d$. Thus the codimension of $\hat{B}_{1}(k)$ in $J^{k} S \times \mathbb{R}^{k}$ is also $k p+1-d$, and the codimension of $B_{1}(k)$ in $J^{k} S$ is $k p+1-d-k=k(p-1)+1-d$.

3.3.2. Codim $\left(B_{2}(k), J^{k} S\right)=d+p-1$. Let $x \in X$ and let $J_{x}^{k} S$ be the (typical) fiber of $J^{k} S$ over $x$. Let $V$ be the subset of $J_{x}^{k} S \times \mathbb{R}^{k}$ defined by $\left(j_{x}^{k} \Sigma, u\right) \in V \Longleftrightarrow f(x)+u_{0} g(x)=0$. The set $V$ has codimension $d$ in $J_{x}^{k} S \times \mathbb{R}^{k}$. Let $\mu_{2}$ be the mapping:

$$
\begin{aligned}
\mu_{2}: \quad V & \longrightarrow\left(T_{x}^{*} X\right)^{p} \times \operatorname{End}\left(T_{x} X\right) \\
\left(j_{x}^{k} \Sigma, u\right) & \longmapsto\left(d h(x), T_{X}\left(f(x)+u_{0} g(x)\right)\right) .
\end{aligned}
$$

We see that $\mu_{2}$ is clearly surjective and the typical fiber of $\hat{B}_{2}(k)$ is the pullback by $\mu_{2}$ of the set of unobservable couples $(C, A)$ of $\left(T_{x}^{*} X\right)^{p} \times$ End $\left(T_{x} X\right)$, whose codimension, according to lemma 6.1 , is $p$. Therefore $\operatorname{codim}\left(\hat{B}_{2}(k), J^{k} S \times \mathbb{R}^{k}\right)=d+p$. But $\hat{B}_{2}(k)$ does not depend on the variables $u_{1}, \ldots, u_{k-1}$ and $\operatorname{codim}\left(B_{2}(k), J^{k} S\right)=d+p-1$.

3.3.3. Codim $\left(B_{3}(k), J^{k} S\right) \geq \min \{k(p-1)+1 ; 2 d\}$. First let us define by $\mathrm{O}_{3}$ the subset of $J^{k} S$ of elements that verify $(f(x), g(x)) \neq(0,0)$. The set $O_{3}$ is clearly an open, dense and PSA subbundle of $J^{k} S$. Let $\hat{B}_{3}^{\prime}(k)=$ $\hat{B}_{3}(k) \cap\left(O_{3} \times \mathbb{R}^{k}\right)$ and $B_{3}^{\prime}(k)=B_{3}(k) \cap O_{3}$. Observe that $\left(j_{x}^{k} \Sigma, u\right) \in \hat{B}_{3}^{\prime}(k)$ implies $g(x) \neq 0$.

For $1 \leq \rho \leq d-1$ let us define $\hat{B}_{6}(k, \rho)$ by :

$$
\hat{B}_{6}(k, \rho)=\left\{\left(j_{x}^{k} \Sigma, u\right) \in \hat{B}_{3}^{\prime}(k) ; \quad u_{1}=u_{2}=\cdots=u_{\rho-1}=0 \text { and } u_{\rho} \neq 0\right\}
$$

We may notice that $u_{1}=u_{2}=\cdots=u_{d-1}=0$ implies $\left(j_{x}^{k} \Sigma, u\right) \notin \hat{B}_{3}^{\prime}(k)$ because of the observability of the linearized system, and conclude that:

$$
\hat{B}_{3}^{\prime}(k)=\bigcup_{1 \leq \rho \leq d-1} \hat{B}_{6}(k, \rho) .
$$

For $1 \leq \rho \leq d-1$, both $\hat{B}_{6}(k, \rho)$ and its projection $B_{6}(k, \rho)$ into $O_{3}$ are PSA.

Let $G_{\rho} \subset\left(O_{3} \oplus T X\right) \times \mathbb{R}^{k}$ be the set of elements $\left(j_{x}^{k} \Sigma, \Lambda, u\right)$ such that:

1. $f(x)+u_{0} g(x)=0$

2. $u_{1}=u_{2}=\cdots=u_{\rho-1}=0$

3. the vectors $A^{i} \Lambda, 0 \leq i \leq \rho$ are linearly independant. 
$G_{\rho}$ is a subbundle and a submanifold of $\left(O_{3} \oplus T X\right) \times \mathbb{R}^{k}$; its codimension equals $d+\rho-1$. Let $\mu_{3}$ be the mapping:

$$
\begin{gathered}
\mu_{3}: \quad G_{\rho} \longrightarrow \mathbb{R}^{k p} \\
\left(j_{x}^{k} \Sigma, \Lambda, u\right) \longmapsto T_{X} R_{k, \Sigma}(x, u) \cdot \Lambda .
\end{gathered}
$$

By Lemma 6.3 of the appendix, $\mu_{3}$ is a submersion and $\operatorname{codim}\left(\mu_{3}^{-1}(0), G_{\rho}\right)$ $=k p$. Therefore, $G_{\rho}$ being conical w.r.t. $\Lambda$, the codimension of the projection of $\mu_{3}^{-1}(0)$ in $O_{3} \times \mathbb{R}^{k}$ is $k p+(d+\rho-1)-(d-1)=k p+\rho$.

Let $\left(j_{x}^{k} \Sigma, u\right) \in \hat{B}_{6}(k, \rho)$. There exists $\Lambda \in T_{x} X, \Lambda \neq 0$, such that $T_{X} R_{k, \Sigma}(x, u) . \Lambda=0$. By lemma 6.3 of the appendix, $C A^{i} \Lambda=0$ for $i=0, \ldots, \rho-1$. The pair $(C, A)$ is observable and this implies that the vectors $A^{i} \Lambda, 0 \leq i \leq \rho$ are linearly independant. As a consequence $\left(j_{x}^{k} \Sigma, u\right)$ belongs to the projection of $\mu_{3}^{-1}(0)$ into $O_{3} \times \mathbb{R}^{k}$. Hence codim $\left(\hat{B}_{6}(k, \rho), O_{3} \times \mathbb{R}^{k}\right) \geq$ $k p+\rho$ and $\operatorname{codim}\left(B_{6}(k, \rho), O_{3}\right) \geq k p+\rho-k=k(p-1)+\rho$. Therefore

$$
\operatorname{Codim}\left(B_{3}^{\prime}(k), J^{k} S\right) \geq \min _{1 \leq \rho \leq d-1}\{k(p-1)+\rho\}=k(p-1)+1 .
$$

Now $B_{3}(k) \backslash B_{3}^{\prime}(k)$ is included in $J^{k} S \backslash O_{3}$. The codimension of $B_{3}(k) \backslash B_{3}^{\prime}(k)$ in $J^{k} S$ is therefore larger or equal to $2 d$ and

$$
\operatorname{Codim}\left(B_{3}(k), J^{k} S\right) \geq \min \{k(p-1)+1 ; 2 d\} .
$$

3.3.4. END OF THE PROOF. It is now possible to compute the codimension of $B(k)$. We have

$$
\begin{gathered}
\operatorname{Codim}\left(B(k), J^{k} S\right) \geq \min _{i=1,2,3} \operatorname{Codim}\left(B_{i}(k), J^{k} S\right) \\
\geq \min \{k(p-1)+1-d, d+p-1, k(p-1)+1,2 d\} .
\end{gathered}
$$

For $p \geq 2$ and $k \geq 2 d$, we have $\operatorname{Codim}\left(B(k), J^{k} S\right) \geq d+1$. The set $B(k)$ is PSA and the same is true for the closure $\overline{B(k)}$ of $B(k)$ in $J^{k} S$. Therefore $\overline{B(k)}$ is a finite union of submanifolds of $J^{k} S$ whose codimension is larger or equal to $d+1$. For $r \geq 2 d+1$, the evaluation mapping: $(\Sigma, x) \mapsto j_{x}^{k} \Sigma$ is a $C^{1}$-mapping. Then by Abraham's theorems on transversality (see [1]), the set of systems $\Sigma$ such that $j^{k} \Sigma$ avoids $\overline{B(k)}$ is open and dense in $S^{r}$. The fact that $j^{k} \Sigma$ avoids $B(k)$ implies obviously that $S R_{k, \Sigma}$ is an immersion. This proves Theorem 3.1 for $2 d+1 \leq r<\infty$. Since the $C^{\infty}$-topology is the inductive limit of the $C^{r}$-topologies, the result holds for $r=\infty$.

\subsection{Proof of the theorem 3.2}

Let $\tilde{X}$ stand for $X \times X \backslash \Delta X$ where $\Delta X$ is the diagonal of $X \times X$, and let $\widetilde{J^{k} S}$ be the restriction of $\left(J^{k} S\right)^{\oplus 2}$ to $\widetilde{X}$.

Let $\widehat{B}_{4}(k)$ be the subset of $\widehat{J^{k} S} \times \mathbb{R}$ whose elements $\left(j_{x_{1}}^{k} \Sigma\right.$, $\left.j_{x_{2}}^{k} \Sigma, u_{0}\right)$ verify:

- $f\left(x_{1}\right)+u_{0} g\left(x_{1}\right)=f\left(x_{2}\right)+u_{0} g\left(x_{2}\right)=0$

- $h\left(x_{1}\right)=h\left(x_{2}\right)$.

The codimension of $\widehat{B}_{4}(k)$ in $\widehat{J^{k} S} \times \mathbb{R}$ equals $2 d+p$. Since $\widehat{B}_{4}(k)$ is PSA, the codimension in $\widehat{J^{k} S}$ of its projection $B_{4}(k)$ is $2 d+p-1$.

ESAIM: COCV, OCTOBER 1998, VoL. 3, 345-359 
Let $\widehat{B}_{5}(k)$ be the PSA subbundle of $\widetilde{J^{k} S} \times \mathbb{R}^{k}$ whose elements $\left(j_{x_{1}}^{k} \Sigma, j_{x_{2}}^{k} \Sigma, u\right)$ verify:

- $f\left(x_{1}\right)+u_{0} g\left(x_{1}\right) \neq 0$ or $f\left(x_{2}\right)+u_{0} g\left(x_{2}\right) \neq 0$

- $R_{k, \Sigma}\left(x_{1}, u\right)=R_{k, \Sigma}\left(x_{2}, u\right)$.

Let $O_{5}$ be the open subset of $\widetilde{J^{k} S} \times \mathbb{R}^{k}$ consisting of elements $\left(j_{x_{1}}^{k} \Sigma, j_{x_{2}}^{k} \Sigma, u\right)$ such that $f\left(x_{1}\right)+u_{0} g\left(x_{1}\right) \neq 0$ or $f\left(x_{2}\right)+u_{0} g\left(x_{2}\right) \neq 0$. By Lemma 6.2 of the appendix the mapping:

$$
\begin{aligned}
\mu_{5}: & O_{5} \longrightarrow \mathbb{R}^{k p} \\
\left(j_{x_{1}}^{k} \Sigma, j_{x_{2}}^{k} \Sigma, u\right) & \longmapsto R_{k, \Sigma}\left(x_{2}, u\right)-R_{k, \Sigma}\left(x_{1}, u\right)
\end{aligned}
$$

is a submersion. Clearly $\widehat{B}_{5}(k)$ is equal to $\mu_{5}^{-1}(0)$, and thus its codimension in $O_{5}$, and also in $\widetilde{J^{k} S} \times \mathbb{R}^{k}$, is $k p$. Finally, the codimension in $\widetilde{J^{k} S}$ of the projection $B_{5}(k)$ of $\widehat{B}_{5}(k)$ is $k p-k=k(p-1)$.

Let $B^{\prime}(k)=B_{4}(k) \cup B_{5}(k)$. The codimension of $B^{\prime}(k)$ is larger or equal to $2 d+1$ as soon as $p \geq 2$ and $k \geq 2 d+1$. By Abraham's theorem on density [1], the set of systems $\Sigma$ such that $\left(j_{x_{1}}^{k} \Sigma, j_{x_{2}}^{k} \Sigma\right)$ avoids $B^{\prime}(k)$ for any $\left(x_{1}, x_{2}\right) \in \widetilde{X}$ is residual (hence dense) in $S^{r}$ for $r \geq 2 d+2$. Thus Theorem 3.2 is proved for $2 d+2 \leq r<\infty$. Since the $C^{\infty}$-topology is the inductive limit of the $C^{r}$-topologies, the result holds for $r=\infty$.

To finish, let $K$ be a compact subset of $\widetilde{X}=X \times X \backslash \Delta X$. Since $B^{\prime}(k)$ is PSA, its closure $\overline{B^{\prime}(k)}$ is again a PSA subbundle of $\widehat{J^{k} S}$ of codimension larger or equal to $2 d+1$ as soon as $p \geq 2$ and $k \geq 2 d+1$. By Abraham's theorem on openness of non-intersection, we can state:

LEMMA 3.4. The set of systems $\Sigma$ such that the mapping:

$$
\begin{aligned}
& K \times \mathbb{R}^{k} \longrightarrow \mathbb{R}^{k p} \\
& \left(x_{1}, x_{2}, u\right) \longmapsto R_{k, \Sigma}\left(x_{2}, u\right)-R_{k, \Sigma}\left(x_{1}, u\right)
\end{aligned}
$$

does not vanish at any point is open and dense in $S^{r}$ for $r \geq 2 d+2, k \geq 2 d+1$ and $p \geq 2$.

This lemma will be used in Section 5 .

\subsection{Proof of the theorem 3.3}

The mapping:

$$
\begin{aligned}
& S^{r} \longrightarrow C^{r-2 d}\left(X \times\left(I_{B}\right)^{2 d} ; \mathbb{R}^{(2 d+1) p}\right) \\
& \Sigma \longmapsto S R_{2 d+1, \Sigma}
\end{aligned}
$$

being continuous, and the set of embeddings being open in $C^{r-2 d}\left(X \times\left(I_{B}\right)^{2 d}\right.$; $\mathbb{R}^{(2 d+1) p)}$ for $p \geq 2$ and $r>2 d$, the set of systems $\Sigma$ such that the restriction of $S R_{2 d+1, \Sigma}$ to $X \times\left(I_{B}\right)^{2 d}$ is an embedding is open in $S^{r}$ (for $r$ large enough); it is dense by Theorem 3.2 and thus Theorem 3.3 is a straightforward consequence of the previous ones. 


\section{THE ANALYTIC CASE}

We consider in this section the set $S^{\omega}$ of real-analytic systems of the form (2.1):

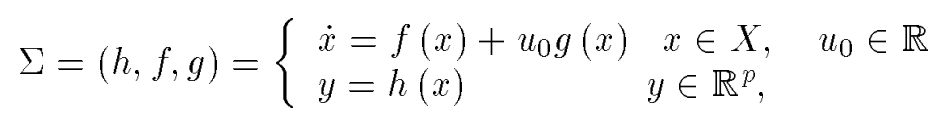

i.e., we assume that $X, h, f$ and $g$ are analytic.

For any system $\Sigma_{0}=\left(h_{0}, f_{0}, g_{0}\right) \in S^{\omega}$, one can build Banach spaces of analytic functions (resp. analytic vector-fields), (resp. analytic systems) $H$, $F, G$ and $S=H \times F \times G$, containing respectively $h_{0}, f_{0}, g_{0}$ and $\Sigma_{0}$ and such that:

1. the norm of $H$ (resp. $F, G, S$ ) is stronger that the norm of $H^{r}$ (resp. $\left.F^{r}, G^{r}, S^{r}\right)$ for $r \in \mathbb{N}$

2. for any distinct points $x_{1}$ and $x_{2}$ in $X$, and for any integer $k$, there exists an element in $H$ (resp. $F, G, S$ ) whose $k$-jets at $x_{1}$ and $x_{2}$ have arbitrary prescribed values.

The proof of the existence of such Banach spaces can be found in [7].

Now, replacing $S^{r}$ by $S$, we can exactly repeat the statements and proofs of Theorems 3.1, 3.2 and 3.3. Thus these theorems remain true in the analytic case.

\section{OBservability For any POLYNOMIAL INPUT With BOUNDED DEGREE}

In this section we examine systems that are observable for any polynomial input whose degree is smaller than an arbitrary but given bound. For the sake of clarity we consider the case $p=2$. The main result is:

Theorem 5.1. Let $b$ be a positive integer. The set of systems that are observable for any polynomial input whose degree is smaller or equal to $b$ contains an open and dense subset of $S^{r}$ (for $r \in \mathbb{N} \cup\{\infty, \omega\}$, large enough).

This theorem is a straightforward consequence of Theorem 5.2, whose statement needs the following definition according to the input compactification in Section 5.1.

Let $b \geq 2 d$ be an integer. We denote by $G_{b}$ the subset of $\mathbb{R}^{K}$, where $K=(d-1)(b+1)+1$, whose elements $(u, \bar{u})=\left(u_{0}, \ldots, u_{b} ; u_{b+1}, \ldots, u_{K-1}\right)$ verify:

- either

$$
\max _{0 \leq i \leq b}\left|u_{i}\right| \leq 1
$$

- or

$$
\begin{gathered}
\left|u_{i}\right| \leq[\lambda(u)]^{i+1} \text { for } \quad b+1 \leq i \leq K-1 \\
\text { where } \lambda(u)=\max _{0 \leq i \leq b}\left|u_{i}\right|^{\frac{1}{i+1}}>1
\end{gathered}
$$

We can now state:

THEOREM 5.2. The set of systems $\Sigma$ such that the restriction of $S R_{K, \Sigma}$ to $X \times G_{b}$ is an embedding contains an open and dense subset of $S^{r}$ (for $r \in \mathbb{N} \cup\{\infty, \omega\}$, large enough).

In order to prove this theorem, we will need two lemmas. ESAIM: COCV, OCTOBER 1998, VOL. 3, 345-359 


\subsection{INPUTS COMPACTIFICATION}

For $\Sigma=(h, f, g) \in S^{r}$ and $\lambda \in \mathbb{R}_{+}^{*}=\{x \in \mathbb{R} \mid x>0\}, \Sigma_{\lambda}$ will stand for the system $(h, \lambda f, g)$. For the same positive real $\lambda$ :

- $\Delta_{\lambda}$ is the linear mapping from $\mathbb{R}^{k}$ into $\mathbb{R}^{k}$ defined by:

$$
\Delta_{\lambda} u=\Delta_{\lambda}\left(u_{0}, u_{1}, \ldots, u_{k-1}\right)=\left(\lambda u_{0}, \lambda^{2} u_{1}, \ldots, \lambda^{k} u_{k-1}\right)
$$

- $A_{\lambda}$ is the linear mapping from $\mathbb{R}^{2 k}$ into $\mathbb{R}^{2 k}$ defined by:

$$
A_{\lambda}\left(y_{1}, z_{1} ; y_{2}, z_{2} ; \ldots ; y_{k}, z_{k}\right)=\left(y_{1}, z_{1} ; \lambda y_{2}, \lambda z_{2} ; \ldots ; \lambda^{k-1} y_{k}, \lambda^{k-1} z_{k}\right) \text {. }
$$

Lemma 5.3. For any system $\Sigma \in S^{r}$, any $\lambda \in \mathbb{R}_{+}^{*}$, and any $k \geq 1$ we have

$$
R_{k, \Sigma_{\lambda}}\left(., \Delta_{\lambda}(.)\right)=A_{\lambda} R_{k, \Sigma}(., .) .
$$

Proof. Let $u=\left(u_{0}, u_{1}, \ldots, u_{k-1}\right) \in \mathbb{R}^{k}$ and let $t \longmapsto u(t)$ be a $C^{k-1}$-input that verifies:

$$
\frac{d^{i} u}{d t^{i}}(0)=u_{i} \text { for } i=0, \ldots, k-1 .
$$

Let us denote by $t \longmapsto \phi(t)$ the solution of $\dot{x}=f(x)+u_{0} g(x)$ passing by the initial condition $\phi(0)=x_{0}$ and corresponding to the input $t \longmapsto u(t)$. The trajectory $\phi$ verifies:

$$
\frac{d}{d t}[\phi(\lambda t)]=\lambda[f \circ \phi(\lambda t)+u(\lambda t) g \circ \phi(\lambda t)] .
$$

Therefore $t \longmapsto \phi(\lambda t)$ is the solution of $\dot{x}=\lambda f(x)+u_{0} g(x)$ for the initial condition $x_{0}$ and the input $t \longmapsto \lambda u(\lambda t)$, whose derivatives verify:

$$
\left.\frac{d^{i}}{d t^{i}}[\lambda u(\lambda t)]\right|_{t=0}=\lambda^{i+1} u_{i} \text { for } i=0, \ldots, k-1 \text {. }
$$

Since:

$$
\left.\frac{d^{i}}{d t^{i}}[h \circ \phi(\lambda t)]\right|_{t=0}=\lambda^{i} \frac{d^{i}}{d t^{i}}(h \circ \phi)(0) \text { for } i=0, \ldots, k-1
$$

and according to the remark 2.1(2), we have:

$$
\begin{aligned}
R_{k, \Sigma_{\lambda}}\left(x_{0}, \Delta_{\lambda} u\right) & =\left(h \circ \phi(\lambda t), \frac{d}{d t}[h \circ \phi(\lambda t)], \ldots, \frac{d^{k-1}}{d t^{k-1}}[h \circ \phi(\lambda t)]\right)_{t=0} \\
& =A_{\lambda}\left(h \circ \phi(t), \frac{d}{d t}[h \circ \phi(t)], \ldots, \frac{d^{k-1}}{d t^{k-1}}[h \circ \phi(t)]\right)_{t=0} \\
& =A_{\lambda} R_{k, \Sigma}\left(x_{0}, u\right) .
\end{aligned}
$$

\subsection{A transversality lemma}

LEMMA 5.4. The set $O$ of systems $\Sigma=(h, f, g)$, such that the mapping from $X$ into $\mathbb{R}^{2 d}$

$$
x \longmapsto\left(h, L_{g} h, \ldots, L_{g}^{d-1} h\right)(x)
$$

is an immersion, is open and dense in $S^{r}$ for $r \geq d$. 
This lemma is a standard application of the transversality theorems to the observability theory of uncontrolled systems. Its proof can be found in $[5]$ or in $[13]$.

\subsection{Proof of Theorem 5.2}

Proof. Let $\Re$ be the set of systems $\Sigma$ that verify:

1. $\Sigma$ belongs to the open set $O$ defined in lemma 5.4 .

2. $S R_{2 d+1, \Sigma}$ is an embedding.

3. $\Sigma$ belongs to the interior of the set of systems $\Sigma^{\prime}$ such that $S R_{2 d, \Sigma^{\prime}}$ is an immersion.

The set $\Re$ is residual in $S^{r}$. Let $\Sigma=(h, f, g) \in \Re$. There exists a neighbourhood of $\Sigma$ in $S^{r}$ whose elements $\Sigma^{\prime}$ are such that $S R_{2 d, \Sigma^{\prime}}$ is a proper immersion. In order to prove theorem 5.2, we have to prove the existence of a neighbourhood of $\Sigma$ in $S^{r}$ whose elements $\Sigma^{\prime}$ are such that the $S R_{2 d, \Sigma^{\prime}}$ are one-to-one.

Let $\left(\Sigma^{n}\right)_{n>1}$ be a sequence of systems that converges to $\Sigma$ in $S^{r}$. We have only to show that the restriction of $S R_{K, \Sigma^{n}}$ to $X \times G_{b}$ is one-to-one for $n$ large enough. Let us assume that for $n \geq 1$ one can find:

$$
\begin{aligned}
& x_{1}^{n} \neq x_{2}^{n} \in X \\
& \left(u^{n}, \bar{u}^{n}\right)=\left(u_{0}^{n}, \ldots, u_{b}^{n} ; u_{b+1}^{n}, \ldots, u_{K-1}^{n}\right) \in G_{b},
\end{aligned}
$$

such that $R_{K, \Sigma^{n}}\left(x_{1}^{n},\left(u^{n}, \bar{u}^{n}\right)\right)=R_{K, \Sigma^{n}}\left(x_{2}^{n},\left(u^{n}, \bar{u}^{n}\right)\right)$.

$X$ being compact, we can extract a subsequence such that:

$$
\begin{aligned}
& x_{1}^{n} \underset{n \rightarrow+\infty}{\longrightarrow} x_{1} \\
& x_{2}^{n} \underset{n \rightarrow+\infty}{\longrightarrow} x_{2}
\end{aligned}
$$

According to lemma 3.4 , the equality $x_{1}=x_{2}$ holds: $\left(x_{1}^{n}, x_{2}^{n}\right)$ converges to $\left(x_{1}, x_{2}\right)$ in $X \times X$; the pair $\left(x_{1}, x_{2}\right)$ cannot belong to any compact subset of $X \times X \backslash \Delta X$. Hence $\left(x_{1}, x_{2}\right) \in \Delta X$.

Let us denote by $x$ the point $x=x_{1}=x_{2}$ and choose a local chart centered at $x$. We can assume that $x_{1}^{n}$ and $x_{2}^{n}$ belong to this chart for all $n \geq 1$. Now we can define:

$$
\Lambda_{n}=\frac{1}{\left\|x_{2}^{n}-x_{1}^{n}\right\|}\left(x_{2}^{n}-x_{1}^{n}\right), \quad n \geq 1,
$$

where $\|\cdot\|$ stands for the Euclidian norm in $\mathbb{R}^{d}$; clearly, $\Lambda_{n}$ belongs to the sphere $S^{d-1}$.

Furthermore, theorem 3.3 implies:

$$
\left\|u^{n}\right\|_{\infty}=\operatorname{Max}\left\{\left|u_{o}^{n}\right|, \ldots,\left|u_{b}^{n}\right|\right\} \longrightarrow+\infty .
$$

In order to obtain convergences, we use the compactification of Section 5.1:

$$
\begin{gathered}
\lambda_{n}=\lambda\left(u^{n}\right)=\underset{0 \leq i \leq b}{\operatorname{Max}}\left|u_{i}^{n}\right|^{\frac{1}{i+1}} \\
v_{i}^{n}=\frac{u_{i}^{n}}{\left(\lambda_{n}\right)^{i+1}} \text { for } i=0, \ldots, K-1 \\
\left(v^{n}, \bar{v}^{n}\right)=\left(v_{0}^{n}, \ldots, v_{b}^{n} ; v_{b+1}^{n}, \ldots, v_{K-1}^{n}\right) \\
=\Delta_{\lambda_{n}^{-1}}\left(u^{n}, \bar{u}^{n}\right) .
\end{gathered}
$$


Taking another subsequence, we have the following convergences:

$$
\begin{aligned}
& \lambda_{n} \underset{n \rightarrow+\infty}{\longrightarrow}+\infty \\
& x_{1}^{n}, x_{2}^{n} \underset{n \rightarrow+\infty}{\longrightarrow}(x, x) \in \Delta X \\
& \Lambda_{n} \longrightarrow{ }_{n \rightarrow+\infty}^{\longrightarrow} \Lambda \in S^{d-1} \\
& \left(v^{n}, \bar{v}^{n}\right) \underset{n \rightarrow+\infty}{\longrightarrow}(v, \bar{v}) \\
& \text { where }\|v\|_{\infty}=1 \text { and }\|\bar{v}\|_{\infty} \leq 1
\end{aligned}
$$

Consider now:

$$
\delta^{n}=\frac{1}{\left\|x_{2}^{n}-x_{1}^{n}\right\|}\left[R_{K, \Sigma_{\lambda_{n}^{-1}}^{n}}\left(x_{2}^{n},\left(v^{n}, \bar{v}^{n}\right)\right)-R_{K, \Sigma_{\lambda_{n}^{-1}}^{n}}\left(x_{1}^{n},\left(v^{n}, \bar{v}^{n}\right)\right)\right] .
$$

According to Lemma 5.3:

$$
\delta^{n}=\frac{1}{\left\|x_{2}^{n}-x_{1}^{n}\right\|} A_{\lambda_{n}^{-1}}\left[R_{K, \Sigma^{n}}\left(x_{2}^{n},\left(u^{n}, \bar{u}^{n}\right)\right)-R_{K, \Sigma^{n}}\left(x_{1}^{n},\left(u^{n}, \bar{u}^{n}\right)\right)\right] .
$$

By assumption $\delta^{n}=0$, and hence the same is true for its limit that we denote by:

$$
\lim _{n \rightarrow+\infty} \delta^{n}=\left(L_{0}, L_{1}, \ldots, L_{K-1}\right) \in \mathbb{R}^{2 K} .
$$

Let us denote by $\delta_{k}^{n}$ the $k^{\text {th }}$ double coordinate of $\delta^{n}$. Clearly $\delta_{k}^{n}$ is a polynomial in the $k-1$ first components of $\left(v^{n}, \bar{v}^{n}\right)$ and its coefficients are derivatives of $h$ w.r.t. $\lambda_{n}^{-1} f_{n}$ and $g_{n}$. Since $\lambda_{n}^{-1} \underset{n \rightarrow+\infty}{\longrightarrow} 0$, all the monomials where derivation w.r.t. $\lambda_{n}^{-1} f_{n}$ arises tend to 0 when $n$ tends to infinity. Therefore:

$$
\begin{aligned}
& L_{0}=h^{(0)} \\
& L_{1}=v_{0} h^{(1)} \\
& L_{2}=v_{0}^{2} h^{(2)}+v_{1} h^{(1)} \\
& \cdots \\
& L_{k}=P_{k, k} h^{(k)}+\cdots+P_{k, 1} h^{(1)}
\end{aligned}
$$

where

$$
h^{(l)}=d L_{g}^{l} h(x) \cdot \Lambda, \quad 0 \leq l \leq K-1 .
$$

$L_{k}$ belongs to $\mathbb{R}^{2}$ and $P_{k, l}$ is a polynomial in $v_{0}, v_{1}, \ldots, v_{k-1}$.

Moreover, for $0 \leq k \leq K-1$, we have that $P_{k, l}$ is a sum of monomials of the form:

$$
\zeta v_{0}^{p_{0}} v_{1}^{p_{1}} \cdots v_{k-1}^{p_{k-1}}
$$

where $\zeta \in \mathbb{N}^{*}=\mathbb{N} \backslash\{0\}$ and:

$$
\text { (*) }\left\{\begin{array}{l}
p_{0}+p_{1}+\cdots+p_{k-1}=l \\
p_{0}+2 p_{1}+\cdots+k p_{k-1}=k .
\end{array}\right.
$$

We prove the property $(*)$ by induction. Clearly $L_{0}=h^{(0)}$ and $L_{1}=$ $v_{0} h^{(1)}$. Let us assume the property is true for $L_{k}$. Clearly $L_{k+1}$ is obtained by derivation of $L_{k}$ w.r.t. $v_{0} g$ and $v_{i+1} \frac{\partial}{\partial v_{i}}$ for $0 \leq i \leq k-1$. Let $m=$ 
$\zeta v_{0}^{p_{0}} v_{1}^{p_{1}} \cdots v_{k-1}^{p_{k-1}}$ be a monomial of $P_{k, l}$ where $0 \leq l \leq k-1$. By assumption we have:

$$
\left\{\begin{array}{l}
p_{0}+p_{1}+\cdots+p_{k-1}=l \\
p_{0}+2 p_{1}+\cdots+k p_{k-1}=k
\end{array}\right.
$$

- If $m h^{(l)}$ is derivated w.r.t. $v_{0} g$ we obtain:

$$
L_{v_{0} g}\left(m h^{(l)}\right)=v_{0} m h^{(l+1)},
$$

$v_{0} m=\zeta v_{0}^{p_{0}+1} v_{1}^{p_{1}} v_{2}^{p_{2}} \cdots v_{k-1}^{p_{k}-1}$ is a monomial of $P_{k+1, l+1}$ that verifies:

$$
\left\{\begin{array}{l}
\left(p_{0}+1\right)+p_{1}+\cdots+p_{k-1}=l+1 \\
\left(p_{0}+1\right)+2 p_{1}+\cdots+k p_{k-1}=k+1
\end{array}\right.
$$

- if $m h^{(l)}$ is derivated w.r.t. $v_{i+1} \frac{\partial}{\partial v_{i}}, 0 \leq i \leq k-1$, we obtain (if $p_{i} \geq 1$ ):

$$
v_{i+1} \frac{\partial}{\partial v_{i}}\left(m h^{(l)}\right)=p_{i} \zeta v_{0}^{p_{0}} \cdots v_{i}^{p_{i}-1} v_{i+1}^{p_{i+1}+1} \cdots v_{k-1}^{p_{k-1}} h^{(l)} .
$$

The monomial $p_{i} \zeta v_{0}^{p_{0}} \cdots v_{i}^{p_{i}-1} v_{i+1}^{p_{i+1}+1} \cdots v_{k-1}^{p_{k-1}}$ of $P_{k+1, l}$ verifies:

$$
\left\{\begin{array}{l}
p_{0}+p_{1}+\cdots+\left(p_{i}-1\right)+\left(p_{i+1}+1\right)+\cdots+p_{k-1}=l \\
p_{0}+2 p_{1}+\cdots+(i+1)\left(p_{i}-1\right)+(i+2)\left(p_{i+1}+1\right)+\cdots+k p_{k-1} \\
=k-(i+1)+(i+2)=k+1
\end{array}\right.
$$

Thus the property $(*)$ is true for $L_{k+1}$.

A consequence of this property is the existence of an integer $j, 1 \leq j \leq$ $\frac{k}{l}-1$, such that $p_{j} \neq 0$. Otherwise we would have:

$$
\sum_{0 \leq i \leq k-1}(i+1) p_{i}>\frac{k}{l} l=k .
$$

Let $s, 0 \leq s \leq d-1$, be the smallest integer for which $h^{(s)}=d L_{g}^{s} h(x) \cdot \Lambda \neq$ 0 . Such an $s$ does exist by lemma 5.4. If $s=0$, then $L_{0}=h^{(0)} \neq 0$. Otherwise, let $q, 0 \leq q \leq b$, be the smallest integer for which $v_{q} \neq 0$. Such a $q$ does exist since $\|v\|_{\infty}=1$. We have:

$$
s(q+1) \leq(d-1) \times(b+1) \leq K-1 .
$$

We will show that:

$$
L_{s(q+1)}=\sum_{l=1}^{s(q+1)} P_{s(q+1), l} h^{(l)} \neq 0 .
$$

- if $l<s, h^{(l)}=0$ by assumption, hence $P_{s(q+1), l} h^{(l)}=0$.

- if $l>s$ let us consider a monomial $m$ of $P_{s(q+1), l}$ :

$$
m=\zeta v_{0}^{p_{0}} v_{1}^{p_{1}} \cdots v_{s(q+1)-1}^{p_{s(q+1)-1}} .
$$

There exists $j \leq \frac{s(q+1)}{l}-1<q$ for which $p_{j} \neq 0$. But $j<q$ emplies $v_{j}=0$. Hence $m=0$ and $P_{s(q+1), l}=0$.

- It remains the case $l=s$.

- one of the monomials of $L_{s(q+1), s}$ is $\zeta v_{q}^{s} h^{(s)}, \zeta \neq 0$. It can easily be obtained by suitable derivations of $L_{0}=h^{(0)}$. 
- let $m=\zeta v_{0}^{p_{0}} v_{1}^{p_{1}} \cdots v_{s(q+1)-1}^{p_{s(q+1)-1}}$ be another monomial of $L_{s(q+1), s}$. There exists $j \neq q$ for which $p_{j} \neq 0$. Moreover:

$$
\begin{gathered}
p_{0}+p_{1}+\cdots+p_{q}+\cdots+p_{s(q+1)-1}=s \\
p_{0}+2 p_{1}+\cdots+(q+1) p_{q}+\cdots+s(q+1) p_{s(q+1)-1}=s(q+1) .
\end{gathered}
$$

Hence:

$$
\frac{1}{q+1} p_{0}+\frac{2}{q+1} p_{1}+\cdots+p_{q}+\cdots+s p_{s(q+1)-1}=s .
$$

If $p_{0}=p_{1}=\cdots=p_{q-1}=0$, then on the one hand:

$$
\frac{q+2}{q+1} p_{q+1}+\cdots+s p_{s(q+1)-1}=p_{q+1}+\cdots+p_{s(q+1)-1}
$$

and on the other hand there exists $j, q+1 \leq j \leq s(q+1)-1$, such that $p_{j} \neq 0$. This is impossible, hence one of the $p_{j}$ 's, $0 \leq j \leq q-1$, is positive. Therefore $m=0$, since $v_{j}=0$.

Finally, we have obtained:

$$
L_{s}(q+1)=\zeta v_{q}^{s} h^{(s)} \neq 0
$$

This is in contradiction with the assumptions $L_{k}=0,0 \leq k \leq K-1$, and the theorem is proved.

\section{APPENDIX}

We state here three lemmas that are proved in [7]. They are used in the proofs of our transversality results.

LEMMA 6.1. Let $d$ and $p$ be two positive integers and let $L$ be the set of linear mappings $L=L\left(\mathbb{R}^{d}, \mathbb{R}^{p}\right) \times L\left(\mathbb{R}^{d}, \mathbb{R}^{p}\right)$. An element $(C, A)$ of $L$ is said to be observable if $\bigcap$ KerC $A^{i}=\{0\}$ or, equivalently, if the rank of $\left(C, C A, C A^{2}, \ldots, C A^{d-1}\right)$ is $d$.

The set of unobservable elements of $L$ is an algebraic subset of $L$ of codimension $p$.

In the statement of the two next lemmas the notations are those of the third section.

LeMMA 6.2. Let $\left(j_{x_{0}}^{k} f, j_{x_{0}}^{k} g, u\right) \in\left(J^{k} \Gamma\right)^{\oplus 2} \times \mathbb{R}^{k}$ such that $f\left(x_{0}\right)+u_{0} g\left(x_{0}\right) \neq$ 0 . Then

1. the mapping

$$
\begin{aligned}
& J_{x_{0}}^{k} H \longrightarrow \mathbb{R}^{k p} \\
& j_{x_{0}}^{k} h \longmapsto R_{k, \Sigma}\left(x_{0}, u\right)
\end{aligned}
$$

is linear and surjective.

2. the mapping

$$
\begin{aligned}
& J_{x_{0}}^{k} H \longrightarrow\left(T_{x_{0}}^{*} X\right)^{k p} \\
& j_{x_{0}}^{k} h \longmapsto T_{X} R_{k, \Sigma}\left(x_{0}, u\right)
\end{aligned}
$$

is linear and surjective.

LeMMA 6.3. Let $\left(j_{x_{0}}^{k} f, j_{x_{0}}^{k} g, \Lambda, u\right) \in\left(\left(J^{k} \Gamma\right)^{\oplus 2} \oplus T X\right) \times \mathbb{R}^{k}$ and $\rho \geq 1$ such that: 
- $f\left(x_{0}\right)+u_{0} g\left(x_{0}\right)=0$;

- $f\left(x_{0}\right) \neq 0$ or $g\left(x_{0}\right) \neq 0$ (hence $g\left(x_{0}\right) \neq 0$ );

- $u_{1}=u_{2}=\cdots=u_{\rho-1}=0$ but $u_{\rho} \neq 0$;

the vectors $A^{i} \Lambda, 0 \leq i \leq \rho$, are linearly independant (where $A=$ $T_{X}\left(f\left(x_{0}\right)+u_{0} g\left(x_{0}\right)\right)$.

Then:

1. the mapping

$$
\begin{aligned}
& J_{x_{0}}^{k} H \longrightarrow \mathbb{R}^{k p} \\
& j_{x_{0}}^{k} h \longmapsto T_{X} R_{k, \Sigma}\left(x_{0}, u\right) . \Lambda
\end{aligned}
$$

is linear and surjective.

2. for $0 \leq i<\rho$

$$
d_{X} L_{F k}^{i} h\left(x_{0}, u\right) . \Lambda=\frac{1}{i !} C A^{i} \Lambda
$$

where $C=d h\left(x_{0}\right)$.

We are very grateful to Professor W. Respondek for his collaboration in the reading and criticism of the text.

\section{REFERENCES}

[1] R. Abraham, J. Robbin: Tranversal mappings and flows, W.A. Benjamin, Inc., 1967.

[2] D. Aeyels: Generic observability of differentiable systems, SIAM J. Contr. Opt., 19, 1981, 595-603.

[3] D. Aeyels: Global observability of Morse-Smale systems, J. Diff. Equations, 45, 1982, $1-15$.

[4] J. Bochnak, M. Coste, M-F. Roy: Géométrie algébrique réelle, Ergebnisse der Mathematik, Springer-Verlag, 1987.

[5] J.-P. Gauthier, H. Hammouri, I. Kupka: Observers for non-linear systems, IEEE CDC Conference, december 1991, 1483-1489.

[6] J.-P. Gauthier, I. Kupka: Observability and observers for nonlinear systems, SIAM J. Contr. Opt., 32, 4, 1994, 975-994.

[7] J.-P. Gauthier, I. Kupka: Observability for systems with more outputs than inputs and asymptotic observers, Math. Z. 223, 1996, 47-78.

[8] J.-P. Gauthier, I. Kupka: Genericity of observability and the existence of nonlinear observers, Banach Center publications, 32, Warszawa 1995, 227-244.

[9] M. Golubitsky, V. Guillemin: Stable mappings and their singularities, Graduate texts in mathematics, Springer-Verlag, 1973.

[10] R. Hermann, J. Krener: Nonlinear controlability and observability, IEEE Trans. Automat. Contr., AC-22, 1977, 728-740.

[11] H. Hironaka: Subanalytic sets, in Number theory, Algebraic geometry and Commutative algebra, in honor of Y. Akizuki, Kinokuniya, Tokyo, 1973, 453-493.

[12] M.W. Hirsch: Differential topology, Graduate texts in mathematics, Springer-Verlag, 1976.

[13] Ph. Jouan: Observability of real analytic vector-fields on compact manifolds, Systems Control Lett., 26, 2, Septembre 1995.

[14] Ph. Jouan: Contribution à l'étude de la généricité de l'observabilité des systèmes contrôlés, submitted to C.R. Acad. Sci. Paris.

[15] Ph. Jouan: Singularités des systèmes non linéaires. Observabilité et observateurs, Thèse de l'Université de Rouen, 1995.

[16] Ph. Jouan, J.-P. Gauthier: Finite singularities of nonlinear systems. Output stabilization, observability and observers, Journal of Dynamical and Control Systems, 2, 2, 1996, 255-288. 
[17] S. Lojasiewicz: Triangulation of semi analytic sets, Annal. Sc. Nor. Sup. PISA, 1966, $449-474$.

[18] H.J. Sussmann: Single-input observability of continuous-time systems, Math. Syst. Theory, 12, 1979, 371-393.

[19] F. Takens: Detecting strange attractors in turbulence, in Dynamic Systems and Turbulence, Warwick, 1980, Springer-Verlag, Berlin, 1981, 366-381.

[20] K. Tchon: On solvability of several affine system problems, Systems Contr. Lett., 4, 1984, 373-379. 